\title{
Propagation of Nonlinear Pressure Waves in Blood
}

\author{
A. Elgarayhi, E. K. El-Shewy, Abeer A. Mahmoud, and Ali A. Elhakem
}

Theoretical Physics Group, Faculty of Science, Mansoura University, Mansoura 35516, Egypt

Correspondence should be addressed to E. K. El-Shewy; emadshewy@yahoo.com

Received 2 June 2013; Accepted 11 July 2013

Academic Editors: B. Vasiev and Z. Yu

Copyright (C) 2013 A. Elgarayhi et al. This is an open access article distributed under the Creative Commons Attribution License, which permits unrestricted use, distribution, and reproduction in any medium, provided the original work is properly cited.

\begin{abstract}
The propagation of weakly nonlinear pressure waves in a fluid-filled elastic tube has been investigated. The reductive perturbation method has been employed to derive the Korteweg-de Vries equation for small but finite amplitude. The effect of the final inner radius of the tube $r_{f}$ on the basic properties of the soliton wave was discussed. Moreover, the conditions of stability and the soliton existence via the potential and the corresponding phase portrait were computed. The applicability of the present investigation to flow problems in arteries is discussed.
\end{abstract}

\section{Introduction}

The intermittent ejection of blood from the left ventricle produces pressure waves that flow in the arterial tree. Experimental data found that the flow velocity in blood vessels largely depends on the elastic properties of the vessel wall and they propagate towards the periphery with a characteristic diagram [1]. In arterial mechanics, the propagation of pressure waves in fluid-filled distensible tubes has been theoretically studied by several researchers [2-4]. Experimental observation for the simultaneous changes in amplitude of the pressure waves at five sites from the ascending aorta to the saphenous artery in dogs showed that the pulsatile character of the blood is soliton waves [5]. Yomosa [6] investigated the nonlinear propagation of solitary waves in large blood vessels. He found that the pulse waves of pressure and flow propagating through the arteries can be described as solitary waves excited by cardiac ejections of blood, and the features of the pulse wave such as "peaking" and "steepening" are interpreted in the viewpoint of soliton. Later, R. M. Shoucri and M. M. Shoucri studied the application of the method of characteristics of shock waves in models of blood flow in the Aorta [7]. Recently, Gaik and Demiray [8] treated the arteries as an incompressible prestressed thin walled elastic tube with a stenosis and the blood as a Newtonian fluid with variable viscosity, which vanishes on the arterial wall, and it takes the maximum value at the center of the artery. They studied the propagation of weakly nonlinear waves in the long wave approximation by the use of the perturbation methods [9]. Many authors examined the stability and the soliton existence condition via the potential and the corresponding phase portrait [10-12]. Elwakil et al. [10] studied the propagation of solitary electron acoustic waves in unmagnetized collisionless plasma. They found that, there are saddle and two-center equilibrium state in the phase plane and there is twofinite separatrix going from a saddle returning to it in the phase portrait. The evolution of small but finite-amplitude propagating solitary structures was studied the by means of Korteweg-de Vries equation (KdV). Many authors have been using the $\mathrm{KdV}$ equation to study the properties of solitary waves $[13,14]$. The major topic of this work is to study the propagation of pressure waves in weakly nonlinear waves in a fluid-filled elastic tube. This paper is organized as follows: in Section 2, we present the basic set of fluid equations governing our model. In Section 3, long wave approximation is used to drive $\mathrm{KdV}$ equation, and the solution for $\mathrm{KdV}$ equation is obtained. In Section 4, some discussions and conclusions are given.

\section{Basic Equations}

To drive the equation of motion of the tube, let us consider a circular cylindrical long tube of radius $R_{0}$ with a uniform inner pressure $P_{0}$, let the axial stretch ratio $\lambda_{z}$ and $r_{0}$ be the radius of the cylindrical tube after such a finite static 
deformation. The position vector of a generic point of the tube may be described by the following:

$$
r=\left(r_{0}+u^{*}\right) e_{r}+z^{*} e_{z}, \quad z^{*}=\lambda_{z} Z,
$$

where $u^{*}\left(z^{*}, t^{*}\right), e_{r}, e_{\theta}, e_{z}, z^{*}$ and $Z$ are a finite time dependent radial displacement, unit base vectors in the cylindrical polar coordinates, the spatial coordinate in the intermediate configuration and the axial coordinate of a point in the undeformed configuration, respectively. The axial displacement is neglected in view of the external tethering. The unit tangent vector $t$ to the meridional curve and the unit exterior normal vector $n$ to the deformed membrane are given by

$$
t=\frac{1}{\Lambda}\left(\frac{\partial u^{*}}{\partial z^{*}} e_{r}+e_{z}\right), \quad n=\frac{1}{\Lambda}\left(e_{r}-\frac{\partial u^{*}}{\partial z^{*}} e_{z}\right),
$$

where $\Lambda$ is defined by

$$
\Lambda=\left[1+\left(\frac{\partial u^{*}}{\partial z^{*}}\right)^{2}\right]^{1 / 2} .
$$

The stretches in the axial and circumferential directions may be given as follows:

$$
\lambda_{1}=\lambda_{z} \Lambda, \quad \lambda_{2}=\lambda_{\theta}+\frac{u^{*}}{R_{0}},
$$

where $\lambda_{\theta}=r_{0} / R_{0}$ is the stretch ratio in the circumferential direction after finite static deformation.

Let $F_{1}$ and $F_{2}$ be the membrane forces acting along each unit length of the meridional and circumferential curves of the tube, respectively. The equation of motion of the tube in the radial direction is given by

$$
\begin{aligned}
& \frac{\partial}{\partial z^{*}}\left\{\frac{\mu}{\left[1+\left(\partial u^{*} / \partial z^{*}\right)^{2}\right]^{1 / 2}} \frac{\partial \Sigma}{\partial \lambda_{1}} \frac{\partial u^{*}}{\partial z^{*}}\right\}-\frac{\mu}{\lambda_{z} R_{0}} \frac{\partial \Sigma}{\partial \lambda_{2}} \\
& \quad+\left(\lambda_{\theta}+\frac{u^{*}}{R_{0}}\right) \frac{P^{*}}{H}=\frac{\rho_{0}}{\lambda_{z}} \frac{\partial^{2} u^{*}}{\partial t^{* 2}},
\end{aligned}
$$

where $\mu$ is the shear modulus of the tube material, $\mu \Sigma$ is the strain energy density function, $H$ is the initial tube thickness, $P^{*}$ is the fluid pressure, and $\rho_{0}$ is the mass density of the tube material. In order to complete the field equations one must know the value of the fluid pressure $P^{*}$. Therefore, (5) is to be complemented with the equations governing the blood fluid. Blood is known to be an incompressible nonNewtonian fluid. The main factor for blood to behave like a non-Newtonian fluid is the deformability of red blood cells and the level of cell concentration (hematocrit ratio). When blood flows in arteries the red cells move to the central region of the artery and, thus, the hematocrit ratio is reduced near the arterial wall, where the shear rate is quite high, as can be seen from Poiseuille flow. In other words, experimental observations indicate that when the shear rate is high, blood behaves like a Newtonian fluid. The ratio of the viscous terms to the nonlinear term is $\left(\mu_{v} / \rho_{f}\right)\left(\partial^{2} v^{*} / \partial z^{2}\right) / v^{*}\left(\partial v^{*} / \partial z\right) \approx$ $\mu_{v} / \rho_{f} V^{\prime} T^{\prime} v^{*} \approx O\left(10^{-5}\right)$, considering $\mu_{v}=0.04 \mathrm{p}$ and $\rho_{f}=$ $1.05 \mathrm{~g} / \mathrm{cm}^{3}$. Therefore, the viscous effect in comparison to the nonlinear effect can be neglected. Based on these observations, we assume that blood is an incompressible inviscid fluid whose equations of axially symmetrical motion in the cylindrical polar coordinates are given by

$$
\begin{gathered}
\frac{\partial V_{r}^{*}}{\partial r}+\frac{V_{r}^{*}}{r}+\frac{\partial V_{z^{*}}^{*}}{\partial z^{*}}=0, \\
\frac{\partial V_{r}^{*}}{\partial t^{*}}+V_{r}^{*} \frac{\partial V_{r}^{*}}{\partial r}+V_{z^{*}}^{*} \frac{\partial V_{r}^{*}}{\partial z^{*}}+\frac{1}{\rho_{f}} \frac{\partial \bar{P}}{\partial r}=0, \\
\frac{\partial V_{z^{*}}^{*}}{\partial t^{*}}+V_{r}^{*} \frac{\partial V_{z^{*}}^{*}}{\partial r}+V_{z^{*}}^{*} \frac{\partial V_{z^{*}}^{*}}{\partial z^{*}}+\frac{1}{\rho_{f}} \frac{\partial \bar{P}}{\partial z^{*}}=0,
\end{gathered}
$$

where $V_{r}^{*}$ and $V_{z^{*}}^{*}$ are the fluid velocity components in the radial and axial directions, respectively, $\rho_{f}$ is the mass density of the fluid, and $\bar{P}$ is the fluid pressure function. These field equations must satisfy the following boundary conditions:

$$
\left.V_{r}^{*}\right|_{r=r_{f}}=\frac{\partial u^{*}}{\partial t^{*}}+\left.\frac{\partial u^{*}}{\partial z^{*}} V_{z^{*}}^{*}\right|_{r=r_{f}},\left.\quad \bar{P}\right|_{r=r_{f}}=P^{*}
$$

Here $\bar{P}$ is the fluid pressure function, $\rho_{f}$ is the fluid mass density, and $r_{f}$ is the final inner radius of the tube. where $\lambda_{\theta}=\left(r_{0} / R_{0}\right)$ is the stretch ratio in the circumferential direction after the finite static deformation, where from (5) it is obtained that

$$
\begin{aligned}
P^{*}= & \frac{\rho_{0} R_{0} H}{\lambda_{z}\left(R_{0} \lambda_{\theta}+u^{*}\right)} \frac{\partial^{2} u^{*}}{\partial t^{* 2}}+\frac{\mu H}{\lambda_{z}\left(R_{0} \lambda_{\theta}+u^{*}\right)} \frac{\partial \Sigma}{\partial \lambda_{2}} \\
& -\frac{R_{0} H}{\left(R_{0} \lambda_{\theta}+u^{*}\right)} \frac{\partial}{\partial z^{*}} \\
& \times\left\{\frac{\mu}{\left[1+\left(\partial u^{*} / \partial z^{*}\right)^{2}\right]^{1 / 2}} \frac{\partial \Sigma}{\partial \lambda_{1}} \frac{\partial u^{*}}{\partial z^{*}}\right\} .
\end{aligned}
$$

In general, the strain energy density $\Sigma$ is a function of $\lambda_{1}$ and $\lambda_{2}$. For our purposes, we shall assume that $\Sigma$ is analytic in $\lambda_{1}$ and $\lambda_{2}$ and can be expanded into power series of the following form:

$$
\begin{aligned}
P^{*}= & \frac{\beta_{1} \mu H}{R_{0}^{2}} u^{*}+\frac{\rho_{0} H}{\lambda_{\theta} \lambda_{z}} \frac{\partial^{2} u^{*}}{\partial t^{* 2}}-\alpha_{0} \mu H \frac{\partial^{2} u^{*}}{\partial z^{* 2}}+\frac{\beta_{2} \mu H}{R_{0}^{3}} u^{* 2} \\
& -\frac{\rho_{0} H}{\lambda_{\theta}^{2} \lambda_{z} R_{0}} u^{*} \frac{\partial^{2} u^{*}}{\partial t^{* 2}}-\frac{\alpha_{1} \mu H}{R_{0}}\left(\frac{\partial u^{*}}{\partial z^{*}}\right)^{2} \\
& +\left(\frac{\alpha_{0} \mu H}{\lambda_{\theta} R_{0}}-\frac{2 \alpha_{1} \mu H}{R_{0}}\right) u^{*} \frac{\partial^{2} u^{*}}{\partial z^{* 2}}+\frac{\mu H}{R_{0}} P_{0},
\end{aligned}
$$


where the coefficients $\alpha_{0}, \ldots, \beta_{2}$ are defined by

$$
\begin{gathered}
\alpha_{0}=\left.\frac{1}{\lambda_{\theta} \lambda_{z}} \frac{\partial \Sigma}{\partial \Lambda}\right|_{u^{*}=0}, \\
\beta_{1}=\left.\frac{R_{0}}{\lambda_{\theta} \lambda_{z}}\left(R_{0} \frac{\partial^{2} \Sigma}{\partial u^{* 2}}-\frac{1}{\lambda_{\theta}} \frac{\partial \Sigma}{\partial u^{*}}\right)\right|_{u^{*}=0}, \\
\beta_{2}=\left.\frac{R_{0}^{3}}{2 \lambda_{\theta} \lambda_{z}} \frac{\partial^{3} \Sigma}{\partial u^{* 3}}\right|_{u^{*}=0}-\frac{\beta_{1}}{\lambda_{\theta}}
\end{gathered}
$$

Equations (6)-(9) give sufficient relations to determine the unknowns $u^{*}, V_{r}^{*}, V_{z^{*}}^{*}$, and $\bar{P}$.

\section{Long Wave Approximation}

The reductive perturbation method is used to study the propagation of small but finite amplitude. Let us introduce the following types of stretched coordinates [15]:

$$
\xi=\epsilon^{1 / 2}\left(z^{*}-g t^{*}\right), \quad \tau=\epsilon^{3 / 2} z^{*},
$$

where $\epsilon$ is a small parameter measuring the smallness of nonlinearity, dissipation, and dispersion, $g$ is the phase velocity in the longwave approximation to be determined later. All physical quantities appearing in (6) and (7) are expanded as power series in $\epsilon$ about their equilibrium values as:

$$
\begin{aligned}
V_{r}^{*} & =\sum_{n=1}^{\infty} \epsilon^{1 / 2+n} V_{r}^{*(n)}(\xi, \tau, r), \\
V_{z^{*}}^{*} & =\sum_{n=1}^{\infty} \epsilon^{n} V_{z^{*}}^{*(n)}(\xi, \tau, r), \\
\bar{P} & =\sum_{n=1}^{\infty} \epsilon^{n} \bar{P}_{(n)}(\xi, \tau, r), \\
u^{*} & =\sum_{n=1}^{\infty} \epsilon^{n} u_{n}^{*}(\xi, \tau, r) .
\end{aligned}
$$

We impose the boundary conditions as follows:

$$
\begin{aligned}
\left.\bar{P}\right|_{r=r_{f}}= & \epsilon\left(\frac{\rho_{0} H g^{2}}{\lambda_{\theta} \lambda_{z}}-\alpha_{0} \mu H\right) \frac{\partial^{2} u^{*}}{\partial \xi^{2}} \\
& +\frac{\beta_{1} \mu H}{R_{0}^{2}} u^{*}-\epsilon^{3} \alpha_{0} \mu H \frac{\partial^{2} u^{*}}{\partial \tau^{2}} \\
& -2 \epsilon^{2} \alpha_{0} \mu H \frac{\partial^{2} u^{*}}{\partial \xi \partial \tau}+\frac{\beta_{2} \mu H}{R_{0}^{3}} u^{* 2}-\epsilon \frac{\rho_{0} H g^{2}}{\lambda_{\theta}^{2} \lambda_{z} R_{0}} u^{*} \frac{\partial^{2} u^{*}}{\partial \xi^{2}}
\end{aligned}
$$

$$
\begin{aligned}
& -\frac{\alpha_{1} \mu H}{R_{0}}\left[\epsilon\left(\frac{\partial u^{*}}{\partial \xi}\right)^{2}+2 \epsilon^{2} \frac{\partial u^{*}}{\partial \xi} \frac{\partial u^{*}}{\partial \tau}+\epsilon^{3}\left(\frac{\partial u^{*}}{\partial \tau}\right)^{2}\right] \\
& +\left(\frac{\alpha_{0} \mu H}{\lambda_{\theta} R_{0}}-\frac{2 \alpha_{1} \mu H}{R_{0}}\right) \\
& \times u^{*}\left(\epsilon \frac{\partial^{2} u^{*}}{\partial \xi^{2}}+\epsilon^{3} \frac{\partial^{2} u^{*}}{\partial \tau^{2}}+\epsilon^{2} \frac{\partial^{2} u^{*}}{\partial \xi \partial \tau}\right)+\frac{\mu H}{R_{0}} P_{0} \\
\left.V_{r}^{*}\right|_{r=r_{f}} & =-\epsilon^{1 / 2} g \frac{\partial u^{*}}{\partial \xi}+\left.\epsilon^{1 / 2}\left(\frac{\partial u^{*}}{\partial \xi}+\epsilon \frac{\partial u^{*}}{\partial \tau}\right) V_{z^{*}}^{*}\right|_{r=r_{f}}
\end{aligned}
$$

Substituting (11) and (12) into (6) and (9) and equating coefficients of like powers of $\epsilon$. Then, from the lowest-order equations in $\epsilon$ the following results are obtained:

$$
\begin{gathered}
\frac{1}{\rho_{f}} \frac{\partial \bar{P}_{1}}{\partial r}=0, \\
-g \frac{\partial V_{z^{*}}^{*(1)}}{\partial \xi}+\frac{1}{\rho_{f}} \frac{\partial \bar{P}_{1}}{\partial \xi}=0 \\
\frac{\partial V_{r}^{*(1)}}{\partial r}+\frac{V_{r}^{*(1)}}{r}+\frac{\partial V_{z^{*}}^{*(1)}}{\partial \xi}=0 .
\end{gathered}
$$

And the boundary conditions can be written as follows:

$$
\left.\bar{P}_{1}\right|_{r=r_{f}}=\frac{\beta_{1} \mu H}{R_{0}^{2}} u_{1}^{*},\left.\quad V_{r}^{*(1)}\right|_{r=r_{f}}=-g \frac{\partial u_{1}^{*}}{\partial \xi} .
$$

From the solution of the sets (14) and (15) we obtain that

$$
\begin{gathered}
u_{1}^{*}=U(\xi, \tau), \quad U(\xi, \tau)=\frac{R_{0}^{2}}{\beta_{1} \mu H} \bar{P}_{1}(\xi, \tau), \\
V_{z^{*}}^{*(1)}=\frac{1}{g \rho_{f}} \bar{P}_{1}(\xi, \tau), \quad V_{r}^{*(1)}=-\frac{1}{2 g \rho_{f}} \frac{\partial \bar{P}_{1}(\xi, \tau)}{\partial \xi} r, \\
g^{2}=\frac{\beta_{1} \mu H r_{f}}{2 \rho_{f} R_{0}^{2}},
\end{gathered}
$$

where $U(\xi, \tau)$ is an unknown function whose governing equation will be obtained later and $g$ is the phase velocity in the longwave approximation.

Considering now the coefficients of $O\left(\epsilon^{2}\right)$, we derive with the aid of (16) the following set of equations

$$
\begin{gathered}
\frac{1}{2 \rho_{f}} \frac{\partial^{2} \bar{P}_{1}(\xi, \tau)}{\partial \xi^{2}} r+\frac{1}{\rho_{f}} \frac{\partial \bar{P}_{2}}{\partial r}=0 \\
-g \frac{\partial V_{z^{*}}^{*(2)}}{\partial \xi}+\frac{1}{g^{2} \rho_{f}^{2}} \bar{P}_{1} \frac{\partial \bar{P}_{1}}{\partial \xi}+\frac{1}{\rho_{f}} \frac{\partial \bar{P}_{2}}{\partial \xi}+\frac{1}{\rho_{f}} \frac{\partial \bar{P}_{1}}{\partial \tau}=0 \\
\frac{\partial V_{r}^{*(2)}}{\partial r}+\frac{V_{r}^{*(2)}}{r}+\frac{\partial V_{z^{*}}^{*(2)}}{\partial \xi}+\frac{1}{g \rho_{f}} \frac{\partial \bar{P}_{1}}{\partial \tau}=0 .
\end{gathered}
$$


And the boundary conditions can be written as follows

$$
\begin{aligned}
\left.\bar{P}_{(2)}\right|_{r=r_{f}}= & \left(\frac{\rho_{0} H g^{2}}{\lambda_{\theta} \lambda_{z}}-\alpha_{0} \mu H\right) \frac{R_{0}^{2}}{\beta_{1} \mu H} \frac{\partial^{2} \bar{P}_{1}}{\partial \xi^{2}} \\
& +\frac{\beta_{1} \mu H}{R_{0}^{2}} u_{2}^{*}+\frac{R_{0} \beta_{2}}{\mu H \beta_{1}^{2}}\left(\bar{P}_{1}\right)^{2}, \\
\left.V_{r}^{*(2)}\right|_{r=r_{f}}= & -g \frac{\partial u_{2}^{*}}{\partial \xi}+\left.\frac{R_{0}^{2}}{g \rho_{f} \beta_{1} \mu H} \bar{P}_{1} \frac{\partial \bar{P}_{1}}{\partial \xi}\right|_{r=r_{f}} .
\end{aligned}
$$

From (17-a) we have

$$
\bar{P}_{2}=-\frac{1}{4} \frac{\partial^{2} \bar{P}_{1}}{\partial \xi^{2}} r^{2}
$$

Eliminating the second order perturbed quantities $\bar{P}_{2}$ and $V_{z^{*}}^{*(2)}$, the desired $\mathrm{KdV}$ equation is obtained as follows:

$$
\frac{\partial \bar{P}_{1}}{\partial \tau}+A \bar{P}_{1} \frac{\partial \bar{P}_{1}}{\partial \xi}+B \frac{\partial^{3} \bar{P}_{1}}{\partial \xi^{3}}=0,
$$

where the coefficients $A$ and $B$ are defined by

$$
\begin{gathered}
A=\frac{R_{0}^{2}}{\beta_{1} \mu H}\left(\frac{\beta_{1} \mu H}{2 g^{2} \rho_{f} R_{0}^{2}}+\frac{2 g^{2} \rho_{f} \beta_{2} R_{0}}{\beta_{1}^{2} \mu H r_{f}}+\frac{1}{r_{f}}\right), \\
B=\left(\frac{g^{2} \rho_{f} R_{0}^{2} r_{f}}{4 \beta_{1} \mu H}+\frac{\rho_{0} \rho_{f} g^{4} R_{0}^{4}}{\lambda_{\theta} \lambda_{z} \beta_{1}^{2} \mu^{2} H r_{f}}-\frac{r_{f}^{2}}{16}-\frac{\alpha_{0} g^{2} R_{0}^{4} \rho_{f}}{\beta_{1}^{2} \mu H r_{f}}\right) .
\end{gathered}
$$

Our system can support two kinds of potential structure depending on the sign of the coefficient of the nonlinear term (A). A stationary solitary wave solution of the KdV equation can be obtained by transforming the space variable to

$$
\eta=(\zeta-v \tau)
$$

where $v$ is velocity of the wave. This has been done by imposing the boundary conditions for localized perturbations, namely, $\bar{P}_{1}=0, d \bar{P}_{1} / d \eta=0$, and $d^{2} \bar{P}_{1} / d \eta^{2}=0$ for $\eta \rightarrow \pm \infty$. Thus, the steady state solution of (22) can be expressed as

$$
\bar{P}_{1}=\bar{P}_{0} \operatorname{sech}^{2}\left[\frac{\eta}{\Delta}\right]
$$

where the soliton amplitude $\bar{P}_{0}$ and the soliton width $\Delta$ are given by

$$
\bar{P}_{0}=\frac{3 v}{A}, \quad \Delta=2 \sqrt{\frac{B}{v}} .
$$

\section{Numerical Results and Discussion}

The weakly nonlinear pressure waves in a fluid-filled elastic tube have been investigated. To make our result physically relevant, numerical studies have been made using parameters close to those values corresponding to actual biologically relevant parameters for experimental data in dogs $[6,16]$.

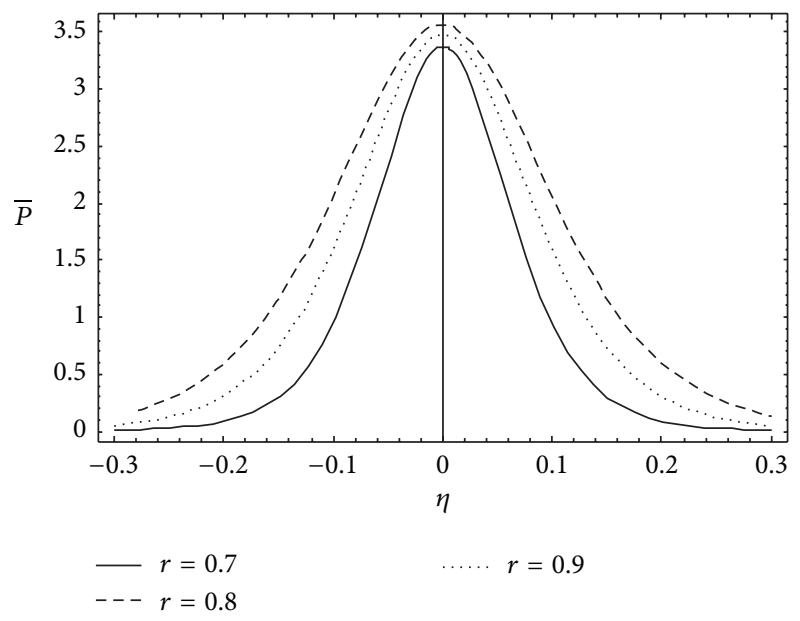

FIgURE 1: The soliton profile for expression (23) for different values of $r_{f}$ for $\beta_{1}=296.105, \beta_{2}=991.496, \rho_{f}=1.05 \mathrm{gm} / \mathrm{cm}^{3}, R_{0}=$ $0.38 \mathrm{~cm}, \rho_{0}=1.03 \mathrm{gm} / \mathrm{cm}^{3}, \lambda_{z}=\lambda_{\theta}=1.6, \alpha_{0}=78.692, H=$ $2 \times 10^{-2} \mathrm{~cm}, \mu=0.4$, and $v=8 \mathrm{~cm} / \mathrm{s}$.

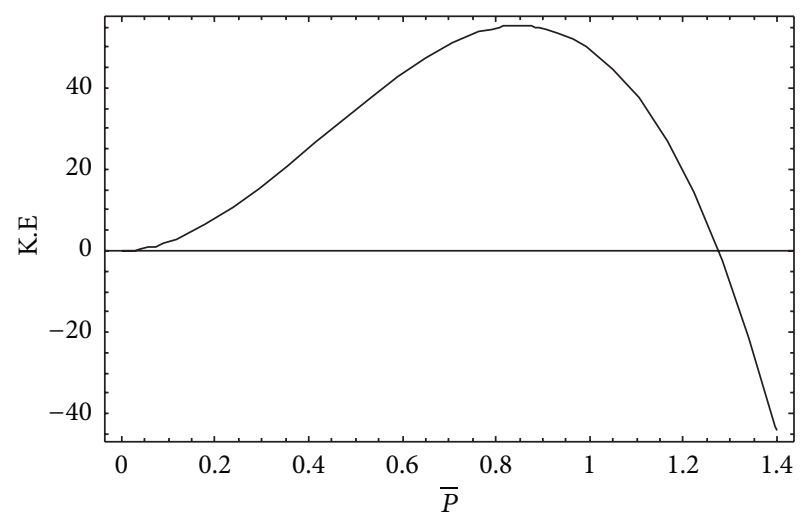

FIgURE 2: The kinetic energy for $r_{f}=0.75, \beta_{1}=296.105, \beta_{2}=$ 991.496, $\rho_{f}=1.05 \mathrm{gm} / \mathrm{cm}^{3}, R_{0}=0.38 \mathrm{~cm}, \rho_{0}=1.03 \mathrm{gm} / \mathrm{cm}^{3}, \lambda_{z}=$ $\lambda_{\theta}=1.6, \alpha_{0}=78.692, H=2 \times 10^{-2} \mathrm{~cm}, \mu=0.4$, and $v=8 \mathrm{~cm} / \mathrm{s}$.

The effect of the final inner radius of the tube $r_{f}$ on the basic properties of the amplitude and width of the soliton is shown in Figure 1. It is obvious that the magnitude of the soliton amplitude and width increases with $r_{f}$. From (20), the nonlinear equation of motion can be obtained as

$$
\frac{1}{2}\left[\frac{d \bar{P}_{1}}{d \eta}\right]^{2}+V\left(\bar{P}_{1}\right)=0 .
$$

This equation can be regarded as an "energy integral" of an oscillating particle of unit mass, with a velocity $d \bar{P}_{1} / d \eta$ and position $\bar{P}_{1}$ in a potential

$$
V\left(\bar{P}_{1}\right)=\left[\frac{\bar{P}_{1}}{6 B}-\frac{v}{2 B}\right] \bar{P}_{1}^{2} .
$$

The kinetic energy of oscillating particle is shown in Figure 2. A necessary condition for the existence of the 

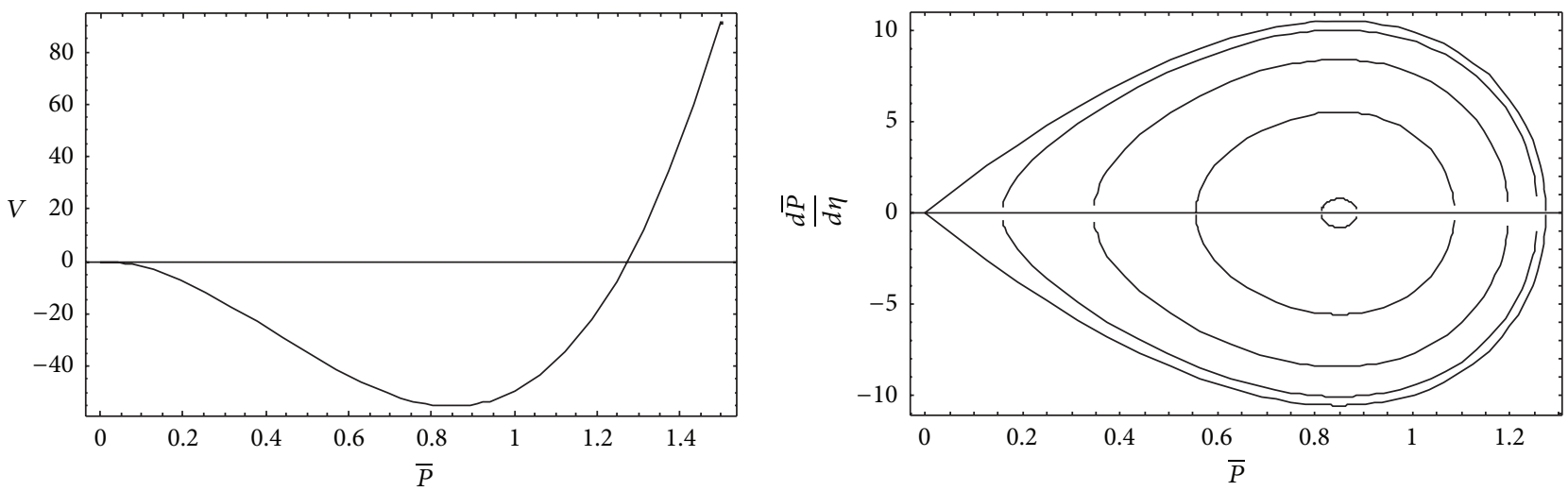

FIGURE 3: The potential and the corresponding phase portrait for $r_{f}=0.75, \beta_{1}=296.105, \beta_{2}=991.496, \rho_{f}=1.05 \mathrm{gm} / \mathrm{cm}^{3}, R_{0}=$ $0.38 \mathrm{~cm}, \rho_{0}=1.03 \mathrm{gm} / \mathrm{cm}^{3}, \lambda_{z}=\lambda_{\theta}=1.6, \alpha_{0}=78.692, H=2 \times 10^{-2} \mathrm{~cm}, \mu=0.4$, and $v=8 \mathrm{~cm} / \mathrm{s}$.

solitary waves is that $d^{2} V / d \bar{P}_{1}^{2}<0$ for $\bar{P}_{1}=0$. A value of $d^{2} V / d \bar{P}_{1}^{2}>0$ predicts the formation of a shock wave. The potential and the corresponding phase portrait are shown in Figure 3. Stable solitons will exist when $v / 2 B>0$; otherwise stable solitons will not be present. The application of our model might be particularly interesting in the new observations for the biological experimental data.

\section{References}

[1] S. C. Ling and H. B. Atabek, "A nonlinear analysis of pulsatile flow in arteries," Journal of Fluid Mechanics, vol. 55, pp. 492-511, 1972.

[2] T. J. Pedley, The Fluid Mechanics of Large Blood Vessels, Cambridge University Press, Cambridge, UK, 1980.

[3] Y. C. Fung, Biodynamics: Circulation, Springer, New York, NY, USA, 1981.

[4] H. Demiray, "Solitary waves in fluid-filled elastic tubes: weakly dispersive case," International Journal of Engineering Science, vol. 39, pp. 439-451, 2000.

[5] D. A. McDonald, Blood Flow in Arteries, Edward Arnold, London, UK, 2nd edition, 1974.

[6] S. Yomosa, "Solitary waves in large blood vessels," Journal of the Physical Society of Japan, vol. 56, pp. 506-520, 1987.

[7] R. M. Shoucri and M. M. Shoucri, "Application of the method of characteristics for the study of shock waves in models of blood flow in the aorta," Cardiovascular Engineering, vol. 7, no. 1, pp. $1-6,2007$.

[8] T. K. Gaik and H. Demiray, "Forced Korteweg-de Vries-Burgers equation in an elastic tube filled with a variable viscosity fluid," Chaos, Solitons and Fractals, vol. 38, no. 4, pp. 1134-1145, 2008.

[9] A. Jeffrey and T. Kawahara, Asymptotic Methods in Nonlinear Wave Theory, Pitman, Boston, Mass, USA, 1981.

[10] S. A. Elwakil, E. K. El-Shewy, and M. A. Zahran, "Higherorder solution of an electron acoustic solitary waves via vortex electron distribution," Chaos, Solitons and Fractals, vol. 22, no. 1, pp. 13-24, 2004.

[11] E. K. El-Shewy, "Higher-order solution of an electron-acoustic solitary waves with non-thermal electrons," Chaos, Solitons \& Fractals, vol. 34, pp. 628-638, 2007.
[12] E. K. El-Shewy, M. I. Abo el Maaty, H. G. Abdelwahed, and M. A. Elmessary, "Solitary solution and energy for the KadomstevPetviashvili equation in two temperatures charged dusty grains," Astrophysics and Space Science, vol. 332, no. 1, pp. 179-186, 2011.

[13] H. R. Pakzad and M. Tribeche, "Electron-acoustic solitons in plasma with nonthermal electrons," Astrophysics and Space Science, vol. 330, no. 1-2, pp. 95-99, 2010.

[14] S. Tasnim, A. Mannan, and A. A. Mamun, "Electrostatic solitary structures in a four-component adiabatic dusty plasma," Astrophysics and Space Science, vol. 337, no. 1, pp. 261-267, 2012.

[15] H. Washimi and T. Taniuti, "Propagation of ion-acoustic solitary waves of small amplitude," Physical Review Letters, vol. 17, no. 19, pp. 996-998, 1966.

[16] H. Demiray, "Solitary waves in prestressed elastic tubes," Bulletin of Mathematical Biology, vol. 58, pp. 939-955, 1996. 

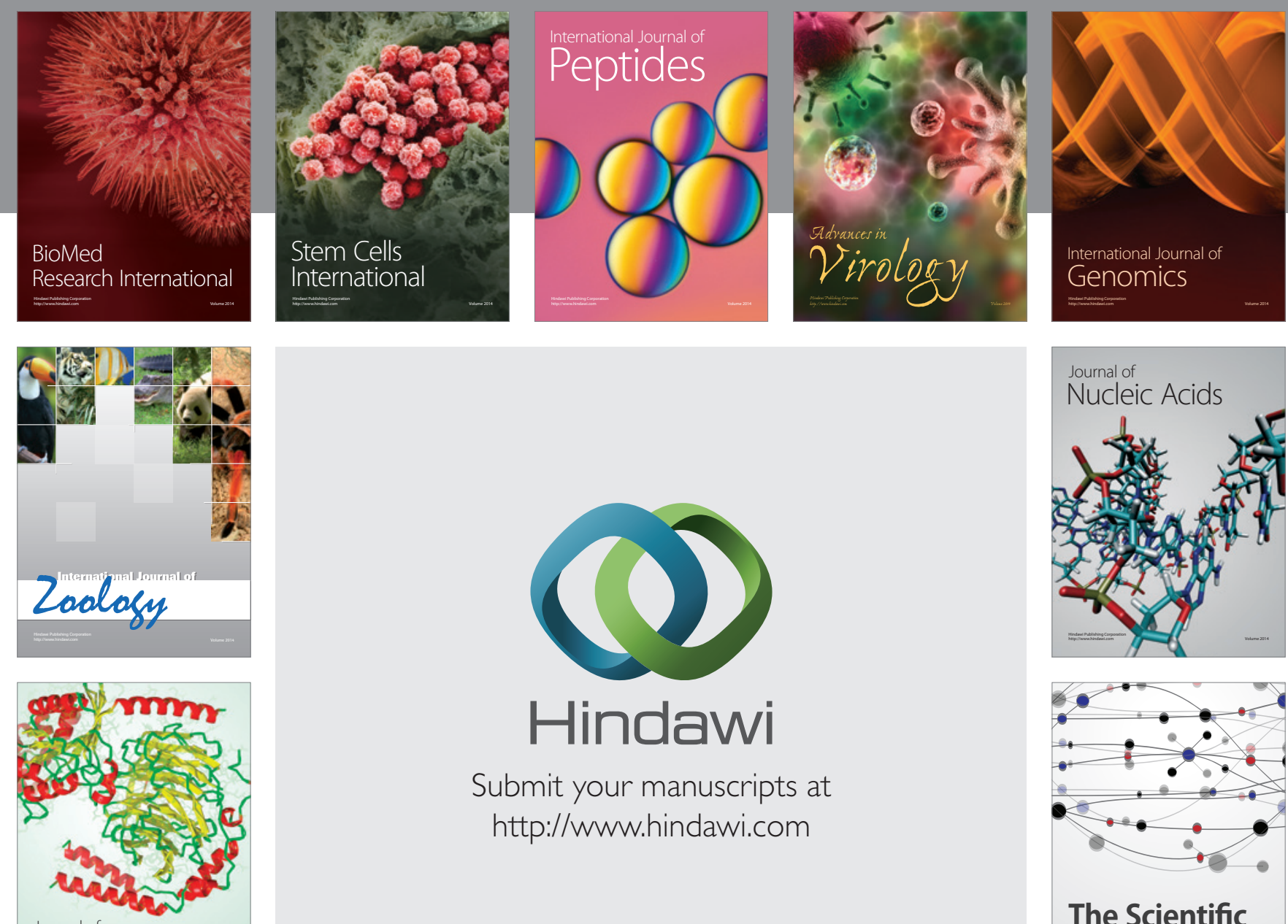

Submit your manuscripts at

http://www.hindawi.com

Journal of
Signal Transduction
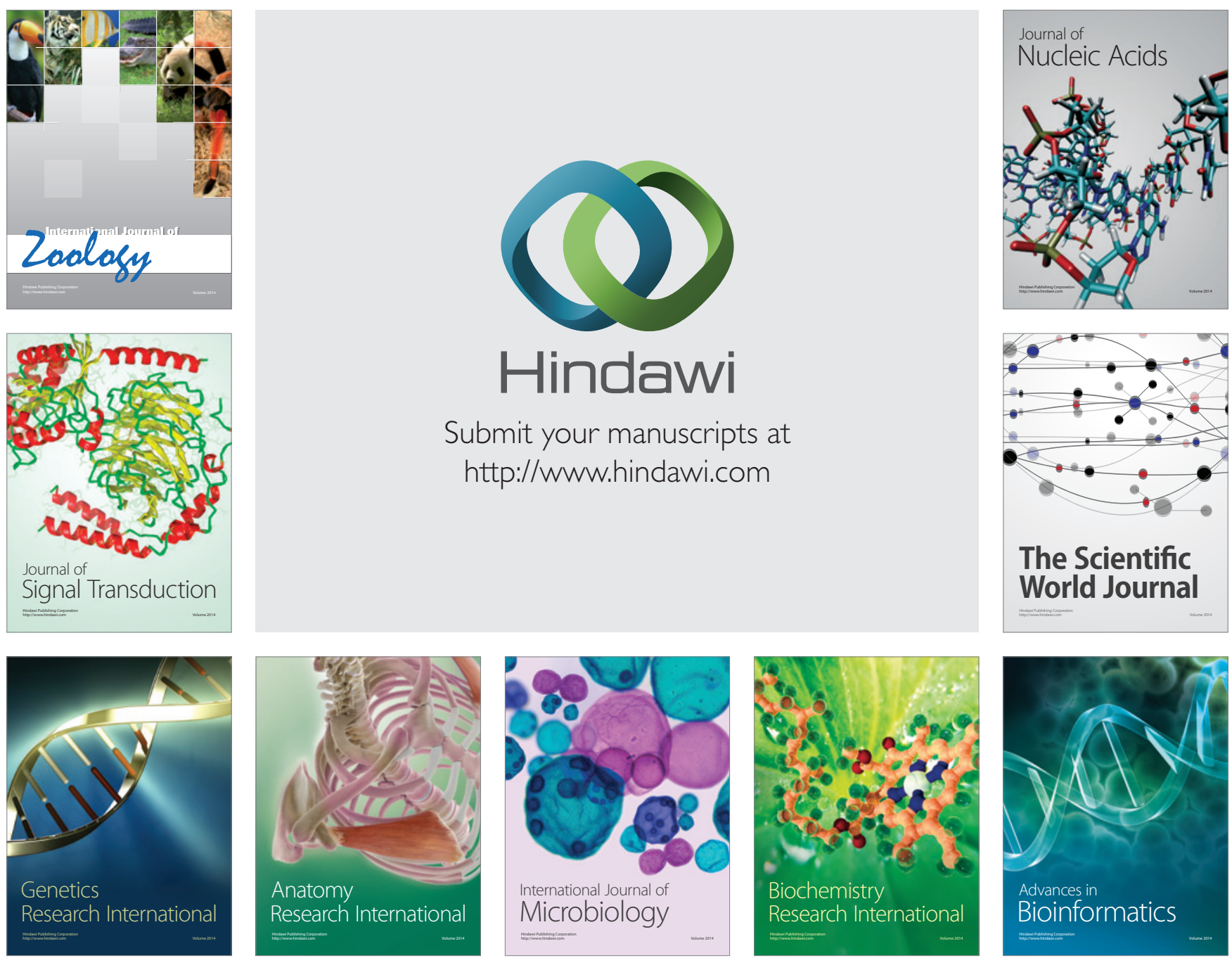

The Scientific World Journal
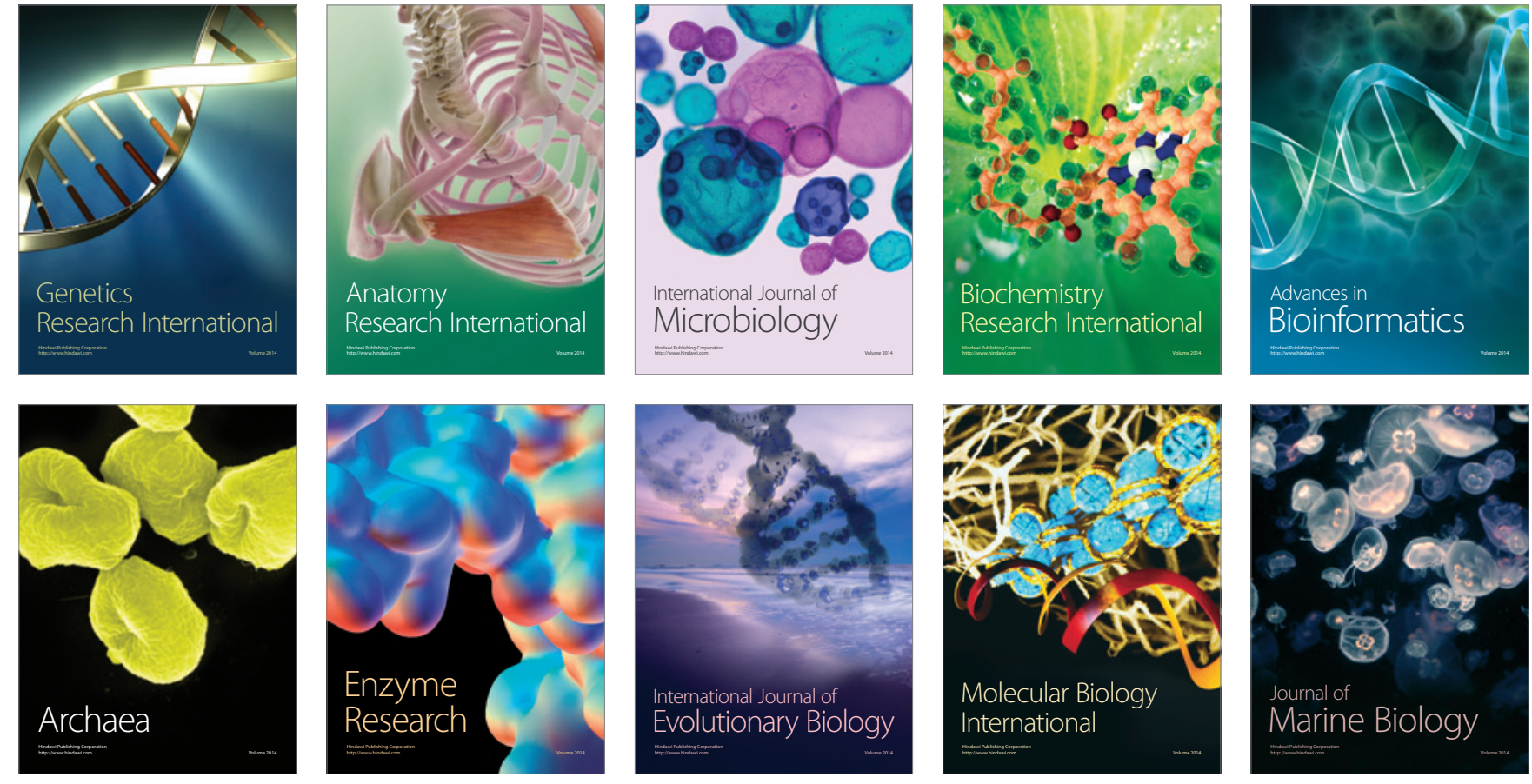